\title{
Neue Anforderungen an die Informationsinfrastruktur von Hochschulen - Dienstestruktur und Serviceentwicklung des IKMZ der BTU Cottbus
}

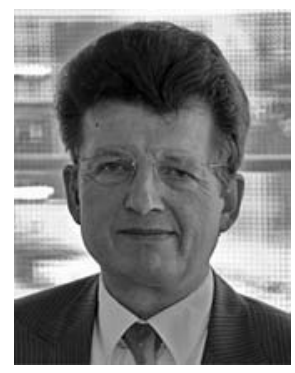

In Anbetracht der starken Marktposition und Wettbewerbsfähigkeit anglo-amerikanischer Universitäten, die dem deutschen Hochschulsystem weit überlegen zu sein scheinen, stellt sich die Frage, welche Herausforderungen an deutschen Hochschulen möglicherweise zu wenig angenommen und umgesetzt werden, um sich im nationalen und internationalen Wettbewerb besser zu positionieren. Die Frage stellt sich auch für Bibliotheken, Medien- und Rechenzentren deutscher Universitäten, deren Beitrag zur Gewährleistung wettbewerbsfähiger Studienbedingungen in Anbetracht des zunehmenden Kostendrucks immer mehr in Frage gestellt wird. Am Beispiel des Informations-, Kommunikations- und Medienzentrums (IKMZ) der Brandenburgischen Technischen Universität Cottbus (BTU Cottbus) wird der dort angelaufene Versuch aufgezeigt, sich als integrierte Einrichtung mit Diensten und Services zu positionieren, die den Forschungs- und Lehrbereich der Hochschule spürbar entlasten und auf diese Weise erfolgreich an Akzeptanz gewinnen.

New requests for the information infrastructure of universities - service structure and development of the IKMZ of BTU Cottbus

On the market for higher education the competitiveness and the strong position of British and Amercian universities seem to be superior to German universities. Which challenges German universities are accepting or picking up to little for improving their position on the national and international market place? From the background of the increasing of costs the question has to be refered also to libraries, computer- and media-centers of German universities, because their ability to improve the conditions for learning and teaching is becoming more and more doubted. The Information-, Communication- and Media Center (IKMZ/ICMC) of the Brandenburg Technical University of Cottbus (BTU Cottbus) is given as an example to relieve the departments with integrated services for education and research substantially and to get the acceptance of the university stakeholders with better success.

Nouvelles exigences à l'infrastructure d'information et de communication des universités - service, structure et développement du IKMZ de l'université technique de Brandebourg à Cottbus

Sur le plan de l'einseignement supérieur la compétitivité et la position forte des universités britanniques et américaines semblent plus élevées qu'aux universités allemandes. Quelles exigences les universités allemandes acceptent-elles, font-elles trop peu pour améliorer leur position sur la place du marché national et international? Sur l'arrière-plan des augmentations des frais la question concerne également les bibliothèques, les centres d'ordinateurs et de médias des universités allemandes parce que leurs facultés d'améliorer les conditions d'étude et d'enseignement se trouvent de plus en plus mises en doute. Le Centre d'information, de communication et de média (IKMZ) de l'université technique de Brandebourg à Cottbus est présenté comme un exemple soutenant les départements avec des services intégrés d'éducation et de recherche et augmentant l'acceptance par les autorités universitaires par un meilleur succès.

\section{$1 \quad$ Aktuelle Herausforderungen}

Als zunehmend problematisch erweist sich für Bibliotheken und Rechenzentren deutscher Hochschulen, die Erfolgsfaktoren ihrer Akzeptanz und Legitimation primär mit einer wissenschaftlichen Ausrichtung zu begründen. Dieser Anspruch lässt sich mit den Anforderungen und Erwartungen an Kundenorientierung und Routinedienste immer weniger in Übereinstimmung bringen. Die Mehrzahl der deutschen Universitätsbibliotheken zählt zu ihren Alleinstellungsmerkmalen weiterhin einen möglichst vollständigen Vorort-Bestand an wissenschaftlichen Monographien und Zeitschriften sowie eine intensive (oft noch lokal spezifische) Formalerschließung der vorhandenen Ressourcen. Dabei ist offenbar der Grundsatz leitend, dass ein Optimum an regelwerksgerechter Verzeichnung die beste Voraussetzung für die Zugänglichkeit der lokalen Bestände ist. Auch die Rechenzentren fühlen sich vielfach einem wissenschaftlichen Anspruch verpflichtet, der am ehesten noch auf dem Gebiet des wissenschaftlichen Rechnens seine Berechtigung hat. Doch nach der längst vollzogenen Ablösung zentraler Mainframe-Systeme durch Client-Server-Anwendungen auf der Basis von Standardsystemen, gängiger Software und Internet-Technologien stehen für den Endnutzer eher Verfügbarkeit von Netzund Systemressourcen und ein daran geknüpfter Support im Vordergrund als die Bereitstellung primär technologiegetriebener (Eigen-)Entwicklungen oder Individuallösungen, die eine nutzerorientierte Handhabung häufig vermissen lassen. Ob beispielsweise der Einsatz komplexer Formatvoriaigentelerr Autdrenp dide-Textersitellangetatsächlich 
erleichtert oder ob dabei nicht eher die Optimierung einer technischen Verarbeitung von Dokumenten im Vordergrund steht, muss nach gegenwärtigem ,state of the art' als offen betrachtet werden.

Ein recht typisches Beispiel für das an deutschen Hochschulen übliche Vorgehen im Innovations- und Weiterentwicklungsbereich ist die Einführung virtueller Lehr- und Lernformen (e-Learning) ${ }^{1}$. Was an amerikanischen und englischen Universitäten seit Mitte der 90ger Jahre primär als Werkzeug zur Optimierung der Lehr-/Lernprozesse verstanden und umgesetzt wird, richtete sich an deutschen Hochschulen in einer Vielzahl von Drittmittel-Projekten zunächst an die Fachbereiche, in denen die Lehre konkret erfolgt. Diese Herangehensweise erscheint unter Bedarfsund Nachfrageaspekten durchaus plausibel; denn wenn es um neue Formen des Lehrens und Lernens geht, sind die Lehrenden sicher primäre Zielgruppe. Um Anwender und Entwickler bei der Lösung technischer Problemstellungen zu entlasten und bei der Aufbereitung von Inhalten mediendidaktisch zu unterstützen, wurden zu den geförderten Vorhaben vielerorts weitere Expertisen in entsprechenden Kompetenzzentren aufgebaut, denen zugleich die Aufgabe der Nachhaltigkeit und der Nachnutzung der entwickelten Modell- oder Pilotlösungen übertragen wurde. Die fast ausschließlich auf Projektbasis erfolgenden Entwicklungen virtueller Lehrformen führten schwerpunktmäßig zu einer wissenschaftlichen Auseinandersetzung mit der Thematik, die einen bemerkenswerten Know-HowZuwachs und Kompetenzgewinn auf diesem Gebiet ergab und fachliche Spezialisierung förderte - allerdings mit der Folge, dass sich das Thema auf Expertenkreise beschränkte und die Hochschulen insgesamt zu wenig erreichte, um neue (virtuelle) Formen des Lehrens und Lernens als strategische Option zu positionieren. Dieses und andere Beispiele machen deutlich, dass es an deutschen Hochschulen häufig zu sehr um einen Wettbewerb geht, bei dem ,the perfect solution of a highly sophisticated problem' im Mittelpunkt steht. Zu wenig konzentriert sich der Wettbewerb auf ,the best practice for a good service' - genau das haben sich die Einrichtungen der Informationsinfrastruktur anglo-amerikanischer Hochschulen zum Ziel gesetzt ${ }^{2}$.

Vor welchen Herausforderungen stehen die deutschen Universitäten und die Einrichtungen ihrer Informationsinfrastruktur? Die Stichworte dafür sind Autonomie, Profilbildung, Wettbewerbsfähigkeit und ein hoher Kostendruck, der alle Bereiche zu überlagern scheint. Die zentralen Infrastrukturbereiche (Bibliothek, Medien- und Rechenzentrum, Verwaltung) sehen sich diesem Kostendruck in besonderer Weise ausgesetzt, weil sie aus Sicht der Hochschulleitung und der Fakultäten oftmals als Kostentreiber beurteilt werden, deren Leistungen keine effizienten Servicemehrwerte erkennen lassen. Eine spürbare Entlastung vermittelt sich Fakultäten und Hochschulleitung in den Diensten und Services von Bibliothek, Medien- und Rechenzentrum (aber auch der Verwaltung) offenbar zu wenig, so dass kürzungsbedingte Einschränkungen das Leistungsangebot der zentralen Infrastrukturbereiche im hochschulinternen Wettbewerb um Akzeptanz und Budgets immer schlechter positionieren - bis hin zu der Einschätzung, dass diese Dienste zwar verfügbar sein sollten, aber keine nennenswerten Kosten verursachen dürfen.

Vor diesem Hintergrund ist unerlässlich, sich im jeweiligen Hochschulkontext als Einrichtung zu positionieren, die weniger in der Erfüllung eines eigenen wissenschaftlichen Anspruchs ihre Rechtfertigung sieht, sondern die ihren Auftrag als Dienst- und Serviceleister vielmehr im Profil der Hochschule und in der Erfüllung der sich daraus ergebenden Anforderungen begründet ${ }^{3}$. So können sich beispielsweise aus der Umstellung auf Bachelor- und Masterstudiengänge Anforderungen an die Attraktivität der Studienbedingungen ergeben, die sich durch eine Optimierung der Informationsinfrastruktur bedienen lassen und zur Aufwertung der daran beteiligten Einrichtungen nachhaltig beitragen. Wenn die dafür vorgesehenen Investitionen plausibel sind, können sich die damit verbundenen Kosten im günstigsten Fall sogar als sekundär erweisen. Allerdings setzt ein solches Vorgehen erkennbare Zielsetzungen der Hochschule und enge Abstimmung mit den Entscheidungsträgern voraus. Für die Einrichtungen der Informationsinfrastruktur bedeutet dies verlässliche Kooperation und Kundenorientierung ${ }^{4}$.

\section{Strukturen und Ziele des IKMZ}

Ob ein solches Vorgehen in Deutschland unmöglich ist? An der Brandenburgischen Technischen Universität Cottbus (BTU Cottbus) wird mit dem Informations-, Kommunikations- und Medienzentrum (IKMZ) jedenfalls der Versuch unternommen, die Informationsinfrastruktur an der absehbaren Entwicklung der Hochschule zu orientieren. Dabei versteht sich die IKMZ-Struktur als eine (jedoch nicht als die einzige) Möglichkeit der Integration der zentralen Bereiche, die auch einen experimentellen, allerdings nicht beliebigen Charakter hat ${ }^{5}$. Gründung und Aufbau des IKMZ stehen in engem Zusammenhang mit einem neuen Gebäude, das Ende 2004 eröffnet wurde und in dem die Bibliothek und das Multimediazentrum untergebracht sind; das Rechenzentrum und die Betriebliche Datenverarbeitung befinden sich an zwei weiteren Standorten auf dem Campus ${ }^{6}$.

Mit Besetzung der Leitungsposition wurde das IKMZ Anfang 2004 als neue zentrale Einrichtung gegründet, in

1 Vgl. auch Lütke-Entrup, Monika; Panke, Stefanie und Guy Tourlemain (2003).

2 Vgl. Cowen, William (1999), Beagle, Donald (1999), Bulpitt, Graham (2002) sowie Virkus, Sirje und Silvi Metsar (2004).

3 Vgl. dazu Simon, ZfBB (2004) und Simon, Positionierung einer Universitäts- und Hochschulbibliothek (2004).

4 Vgl. dazu auch Roosendaal, Hans; Geurts, Peter und Eberhard Hilf (2004).

5 Vgl. auch das im Rahmen der DFG-Förderinitiative geförderte Integrationsvorhaben der Universität Oldenburg unter <http://www.uni-oldenburg.de/projekti3sic/> und das Kommunikations- und Informationszentrum der Universität UIm unter <http://kiz.uni-ulm.de/>. Der Entwicklungsstand im angloamerikanischen Bereich ist von Mel Collier beschrieben worden. Collier, Mel: The context of convergence. In: Oldroyd, M.: Staff development in academic libraries. Present practice and future challenges. London 1996, S. 68-80. Mit einer im Erscheinen begriffenen Publikation wird von Collier die Entwicklung außerhalb Großbritanniens mit Stand 2004 dargestellt.

6 Vgl. dazu Warnatz (2005) und Degkwitz, B.I.T-Online (2005 p̧ereitgestellt von | Humboldt-Universität zu Berlin 
der die bisher weitgehend autarken Bereiche Bibliothek, Multimediazentrum, Rechenzentrum und Betriebliche Datenverarbeitung unter einer gemeinsamen Leitung zusammengefasst sind. Nach dem in 2003 gefassten Gründungsbeschluss von Präsidium und Senat verbinden sich mit der neuen Einrichtung als Zielsetzungen:

- Einrichtung eines übergreifenden Managements mit Gesamtverantwortung für die Informations-, Kommunikations- und Mediendienste der Universität,

- Realisierung effizienter Organisationsstrukturen mit einem breiten Spektrum kunden- und serviceorientierter luK-Angebote,

- Integration der Aufgabenbereiche unter Wahrung der jeweiligen Kernkompetenzen und Optimierung der Dienste und Services,

- Schaffung von Freiräumen für Innovation und nachhaltige Weiterentwicklung durch höhere Kosteneffizienz der Arbeits- und Betriebsabläufe.

Ausgangspunkt für die Entwicklung integrierter Diensteund Servicestrukturen ist eine Funktionsstruktur (s. Abb. 1), in der die Aufgaben- und Funktionsbereiche des IKMZ - losgelöst von den Dienste erbringenden Einrichtungen - modellhaft dargestellt sind. Wie die Übersicht zeigt, handelt es sich einerseits um Aufgabenbereiche, die von Bibliothek, Multimediazentrum, Rechenzentrum und Verwaltungsdatenverarbeitung auf Basis der jeweils einrichtungsbezogenen Kernkompetenz bereits eingebracht werden, und andererseits geht es um die folgenden neuen Funktionsbereiche, deren Gewährleistung auf der organisatorischen und/oder technischen Ebene eine stärkere Integration erfordern oder voraussetzen ${ }^{7}$.

\subsection{Content- und Data-Management}

Dieser neu zu konzipierende Funktionsbereich soll die ,Wissenschaftliche Informationsversorgung', ,MultimediaProduktion und -Publikation' sowie ,Management- und Verwaltungsinformation' stärker vernetzen. Die bisher in getrennten Bereichen erbrachten Dienste und Services sollen durch eng zusammenarbeitende Teams weiterentwickelt werden, um die Mehrwerte einer möglichst weitgehenden Integration der Systeme, zum Aufbau von Schnittstellen und zum Einsatz eines zentralisierten Content- und Datenmanagement-Systems zu nutzen. Dazu gehören Dienste und Services aus allen Bereichen des IKMZ: Bibliothekssystem, Dokumentmanagementsysteme, Identity-Management, Management- und Verwaltungssysteme (HIS), Lernplattformen, Intranet-Informationssysteme, Mediensysteme (z. B. spezielle Videospeicherungssysteme) etc.

\section{$2.2 \quad$ Front-Office}

Mit der Entwicklung des Front-Office verbindet sich das Ziel, die durch die Funktionsbereiche des IKMZ zur Verfügung gestellten Dienstleistungen einrichtungsübergreifend an alle internen und externen Zielgruppen zu kommunizieren und zu vermitteln. Damit versteht sich das Front-Office als ,Nutzerschnittstelle' des IKMZ. Das FrontOffice hat mit seiner direkten Anbindung an jeden der Funktionsbereiche eine Querschnittsfunktion und stellt die Verbindung zwischen den Funktionsbereichen und den Nutzergruppen (Wissenschaftler, Studierende, Ver- waltung, externe Nutzer) her. Durch seine Vernetzung mit allen Funktionsbereichen ermöglicht das Front-Office die Bereitstellung von integrierten, funktionsbereichübergreifenden Dienstleistungen.

Mit dem Front-Office soll im neuen Gebäude ein breites Spektrum allgemeiner Informations- und Kommunikationsdienste konzentriert werden. Dazu gehören insbesondere: Zugriff auf gedruckte und elektronische Medien, Print- und Plotdienste, Medienbearbeitung, Videoconferencing, Computer-Pools, Auskunft, Beratung. Über diese Funktionen im neuen Gebäude hinaus gehören zu den Service-Aufgaben des Front-Office:

- Aufbau stationärer und virtueller Help-Desk-Funktion für Nutzerberatung und -unterstützung,

- Entwicklung eines zentralen IKMZ-Internet-Portals, mit dem alle IKMZ-Dienste- und Serviceleistungen kommuniziert und transparent gemacht werden,

- Bereitstellung in- und externer Weiterbildungsangebote zu allen vom IKMZ abgedeckten Funktionsbereichen (Vermittlung von IT- und Medienkompetenz).

Wesentliche Zielsetzung des Front-Office ist das Angebot integrierter Dienstleistungen „aus einer Hand“ und nach Möglichkeit über jeweils zentrale Ansprechpartner.

\subsection{Innovations-Office}

Das Innovations-Office hat das Ziel, die Forschungs- und Entwicklungsleistungen auf dem Gebiet der Informationsversorgung, des Daten- und Wissensmanagements und der Publikation und Verbreitung von wissenschaftlichen Informationen pragmatisch zu konzipieren und einer raschen und nachhaltigen Umsetzung zuzuführen. Das Innovations-Office versteht sich insofern als Entwicklungsabteilung des IKMZ und bietet den organisatorischen Rahmen, um neue Entwicklungen und Nachfragebereiche flexibel und rasch aufzugreifen und um Experten-KnowHow (auch aus den Fakultäten der Universität) in aktuelle Entwicklungen einzubeziehen.

Das Innovations-Office hat analog zum Front-Office eine Querschnittsfunktion. Auf diese Weise können Aufbau, Implementierung und Vernetzung interdisziplinärer und übergreifender Entwicklungen effektiv vorangetrieben werden. Zu den Aufgaben des Innovationsoffice gehören: Durchführung von innovativen Projekten, Bildung von Projektteams (Drittmittelmitarbeiter, befristet zugeordnete Mitarbeiter aus allen Funktionsbereichen etc.), gemeinsames Projektmanagement, Drittmittelakquisition, Entwicklung und Erprobung neuer Dienste und deren Überführung in den Regelbetrieb.

\section{Chancen und Risiken der Entwicklung}

Ausgehend vom Querschnittscharakter des Frontoffice und des Innovationsoffice setzt die Integration von Diensten und Services einerseits an der ,Nutzerschnittstelle' und andererseits an der ,Entwicklungsschnittstelle' an. In der als Schichtenmodell strukturierten Sicht (s. Abb. 2), die sich als ,Arbeitsthese' für die Weiterentwicklung versteht, bilden die Netz- und Sicherheitsdienste die Grundlage für alle IT-Dienstleistungen, auf der die Rechen- und Sys-

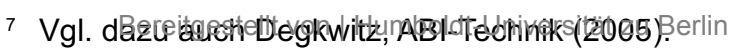


temleistungen als Voraussetzung für den neuen Funktionsbereich ,Content- und Datamanagement' aufbauen. Dieser neue Bereich ist Grundlage und Voraussetzung für die Integration der Funktionen ,Multimedia-Produktion und Publikation', ,Wissenschaftliche Literatur- und Informationsversorgung' und ,Management- und Verwaltungsinformationen'.

Dabei soll der Integrationsansatz einerseits technisch und andererseits organisatorisch vollzogen werden. Die technische Integration bezieht sich insbesondere auf die Funktionen: Archiv- und Back-Up, Homogenität der Stammdaten von BTU-Mitarbeitern und Studierenden, Mail- und WEB-Services, Vereinheitlichung des Einsatzes von Betriebssystemen und Software. Auf diese Weise sollen - im Rahmen des technisch Möglichen und Vertretbaren - Redundanzen im Hard- und Softwarebereich sowie bei der Datenhaltung vermieden und zugleich auf eine nachhaltige Standardisierung von Basisdiensten hingewirkt werden. Die organisatorische Integration greift Dienste und Serviceleistungen auf, in denen sich einzelne Funktionsbereiche entweder überschneiden und/oder an denen mindestens zwei Funktionsbereiche zu beteiligen sind; dazu gehören Authentifizierungs- und Autorisierungsdienste, File- und Storage-Dienste sowie Produktions- und Publikationsdienste. Leitende Zielsetzung ist dabei, den Nutzer- und Zielgruppen des IKMZ möglichst homogene Arbeitsumgebungen als ,workflow-basierte' Serviceangebote zur Verfügung zu stellen.

Die genannten Funktionsbereiche sind organisatorisch im IKMZ zusammengefasst und unter eine gemeinsame Leitung zusammengeführt. Der Leiter des IKMZ hat die Verantwortung für Betrieb und Weiterentwicklung des IKMZ, die auch die Budgetverantwortung (ca. $€ 5$ Mio. in 2004) und die Personalverantwortung für rund 100 Mitarbeiter umfasst; er berichtet dem Präsidenten bzw. Präsidialkollegium der Universität. Zugleich obliegt der IKMZ-Leitung die Verantwortung für Weiterentwicklung und Umsetzung der luK-Strategie der Universität im Einklang mit der Gesamtstrategie der Hochschule. Die zweite Leitungsebene im Rahmen des IKMZ wird durch die Leitungen der einzelnen Teilbereiche des IKMZ (Bibliothek, Multimediazentrum, Rechenzentrum, Verwaltungsdatenverarbeitung) gebildet. Zur Vermeidung von ,Administrations-Overhead' orientiert sich die operationelle Zusammenarbeit der zweiten Leitungsebene - im Sinne der allgemeinen Entwicklungsziele - schwerpunktmäßig auf konkret anstehende Vorhaben, z. B. Drittmittelbeantragung, DV-Ausstattung und Vernetzung des neuen Gebäudes, WEB-Präsenz, Helpdesk- und Serviceoptionen im Rahmen des Front-Office etc. Die fachliche Umsetzung dieser Projekte erfolgt dann in entsprechenden Teams, deren Einsatz in Abhängigkeit von der Projektzielsetzung kurz- oder längerfristig ist.

Ein noch einzusetzender IKMZ-Beirat soll Beteiligungen und Kompetenzen bisher tätiger Ausschüsse und Kommissionen (Bibliotheksausschuss, EDV-Ausschuss, usw.) bündeln und erweitern. Der Beirat soll sich in folgender Weise zusammensetzen: der zuständige Vizepräsident als Vertreter des Präsidialkollegiums (Vorsitz), jeweils ein Vertreter der (vier) Fakultäten, der Kanzler als Vertreter der Verwaltung, ein Vertreter/-In der wiss. Mitarbeiter/-Innen, ein Vertreter/-In der Studierenden. Darüber hinaus sollen als themenbezogene Arbeitsgremien Fokusgruppen gebildet werden, in denen die Vertreter von Nutzer- und Zielgruppen des luK-Angebots und Mitarbeiter der IKMZ zusammenarbeiten, um punktuelle Probleme zu lösen und einzelne Dienste- und Serviceangebote zu verbessern. In Abbildung 3 wird die Einbindung des IKMZ in die Universitätsstruktur verdeutlicht.

\subsection{Diensteentwicklung}

Für den weiteren Auf- und Ausbau des Serviceprofils des IKMZ hat die Umsetzung folgender Ziele im Sinne der Hochschulentwicklung hohe Priorität:

- Helpdesk- und Supportangebote für die Produktion, Nutzung und Verarbeitung von Medien sowie der dafür notwendigen technischen Hilfsmittel (Hotline),

- Ausbau des Schulungs- und Weiterbildungsangebots für Forschende, Lehrende, Studierende und Mitarbeiter zur Steigerung und Verbesserung von Informationskompetenz (Teaching-Library),

- Bereitstellung eines nachfragegerechten Ressourcenangebots an digitalen und gedruckten Inhalten mit einem Schwerpunkt auf elektronischen Materialien (Datenbanken, e-Books, Zeitschriften etc.),

- Aufbau eines Content- und Storage-Managements zur Bereitstellung hochschuleigener Ressourcen (Forschungsergebnisse, Primärdaten, Lehr-/Lernmaterialien, Qualifikationsschriften etc.) mit Ausbau der File- und Back-Up-Dienste (einschl. Archivierung) sowie Weiterentwicklung bestehender Web-basierter Informationsangebote der Universität für in- und externe Informationen,

- Verbesserung der Authentifizierungs- und Zugriffssteuerung (gemeinsame Datenhaltung, Verzeichnisdienste etc.) sowie Vereinfachung der Zugangs- und Zugriffsoptionen über interaktive (personalisierte) Portalfunktionen,

- Bereitstellung von Produktionswerkzeugen für Animationen, Graphik-, Video- und Audiobearbeitung, zur Dokumenterstellung und -konvertierung sowie der Ausbau des Einsatzes von Video-Conferencing,

- signifikante Erhöhung von online-gestütztem Lehren und Lernen durch Bereitstellung und Betreuung von Kurs-Managementsystemen (Lernplattformen) sowie Entwicklung und Erstellung von Lehr-/Lernszenarien zu nachnutzbaren bzw. vermarktbaren Produkten.

Mit der Umsetzung dieser Ziele wird der Tatsache Rechnung getragen, dass computerbasiertes Lernen die Informations- und Medienversorgung in Forschung, Lehre und Studium deutlich verändert hat und weiterhin verändert: Digitale und gedruckte Informationsträger ergänzen sich wechselseitig, dynamische und statische Inhalte greifen verstärkt ineinander. Bereitstellung und Nutzung von Daten, Informationen und Inhalten erfolgen über eine Vielzahl von Arbeitsplattformen, Trägermedien und Zugangskanälen. Für die Produktion, Verbreitung, Nutzung und Verarbeitung von Daten und Inhalten bieten sich vielfältige Formen ${ }^{8}$.

\subsection{Organisationsentwicklung}

Um dieses Spektrum zu gewährleisten und zur Verfügung zu stellen, ergeben sich für Betrieb und Organisation des IKMZ und seiner Teilbereiche folgende Fragen:

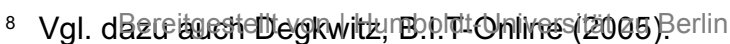


- Wie lässt sich das Dienstleistungsangebot verändern? Welchen Stellenwert wird das bisherige Funktionsspektrum der IKMZ-Teilbereiche künttig haben?

- Welche Ressourcen bzw. Ressourcenzugänge werden in welchem Umfang künftig vorgehalten: Contentressourcen (Monographien, Zeitschriften etc.), Netzwerkressourcen, Rechner- und Systemressourcen, Beratung und Support?

- Welche neuen Aufgaben erfordern welche neuen Organisationskonzepte? Auf welche traditionellen Aufgaben kann verzichtet werden?

- Welche Qualifizierungsanforderungen kommen auf Mitarbeiter und Mitarbeiterinnen zu? Welche neuen Qualifikationen sind erforderlich?

- Welche Finanzierungsmodelle und/oder Geschäftsmodelle zeichnen sich ab? Finanzierung über Leistungsund Zielvereinbarungen oder über rein nutzungsabhängige Modelle?

Insgesamt lässt sich feststellen, dass sich mit neuen Technologien auch neue Serviceerwartungen verbinden und sich insofern ein Paradigmenwechsel abzeichnet, der über den Wechsel der Technologie hinaus tief greifende Veränderungen für das Betriebs- und Managementkonzept hat. Die benannten Fragekomplexe im Sinne der Serviceentwicklung zu beantworten, steht im Mittelpunkt der Weiterentwicklung des IKMZ. In diesem Kontext wird der experimentelle Charakter, aber auch die Dienstleistungsorientierung des Vorgehens deutlich. Es geht weder darum, ein Idealmodell zu realisieren, das schnell zum Selbstzweck wird, noch soll ein bereits ,fertiges' Modell die Nachfrage- und Serviceentwicklung vorwegnehmen, wobei ein Konsens darüber eine Laufzeit erwarten ließe, die im Extremfall mehr Stillstand als Weiterentwicklung zur Folge hätte.

Die Vorteile des eingeschlagenen Weges sind darin zu sehen, dass ein Höchstmaß an Flexibilität innerhalb definierter Rahmenbedingungen gewährleistet ist. Hinzu kommt, dass die bereichsübergreifende Leitung die Informationsinfrastruktur nach innen und außen als Ganzes vertritt, so dass das von der Angebotsseite her angestrebte ,one face to the customer' eine institutionalisierte Entsprechung hat. Als außerordentlich hilfreich wird sich für den begonnenen Change-Management-Prozess das kürzlich bewilligte BMBF-Projekt eLearn@BTU erweisen, mit dem virtuelle Formen des Lehrens und Lernens stärker im Studienangebot der BTU Cottbus verankert werden (e-Learning-Integration) und das sich mit einem eigenen Schwerpunkt auch auf die Weiterentwicklung der Informationsinfrastruktur bezieht.

\subsection{Problem- und Risikofelder}

Natürlich bestehen auch Probleme und Risiken, die sich vor allem auf folgenden Feldern bemerkbar machen":

- Die mit der Optimierung der luK-Strukturen verbundene Kostenentwicklung, der wachsende Finanzierungsbedarfe von anderer Seite (Fakultäten) und damit kontinuierlich enger werdende Finanzierungsspielräume gegenüberstehen,

- die Akzeptanz der unter Aspekten der Kostenkonsolidierung verstärkten Standardisierung von Diensten und Serviceleistungen bis hin zum Verzicht auf Leistungen, die mit dem Profil der Hochschule oder mit ihrer absehbaren Entwicklung nicht mehr zu vereinen sind,
- die Implementierung pragmatischer Verfahren zur Performancemessung und zum Qualitätsmanagement, die sich mit der akademischen Tradition deutscher Universitäten nicht unmittelbar in Übereinstimmung bringen lassen.

Als besonders schwierig erweist sich ein Leistungsverzicht, der die Hochschulentwicklung wegweisend prägen kann. So zeigt ein signifikanter Verzicht auf Subskriptionen wissenschaftlicher Zeitschriften in eine Richtung, die das Servicespektrum der zentralen Informationsinfrastruktur vorrangig im Bereich von Lehre und Studium positioniert. Vergleichbares gilt für den Verzicht auf zentrale Infrastruktur für das wissenschaftliche Rechnen. Über keinerlei Möglichkeiten des virtuellen Lehrens und Lernens zu verfügen, lässt Rückschlüsse auf den Stellenwert von Innovation in Lehre und Studium zu. Auch die mancherorts in Erwägung gezogene Zusammenlegung von IP-Domains kann das Selbstverständnis der daran beteiligten Hochschulen substantiell treffen. Die in diesem Zusammenhang anstehenden Richtungsentscheidungen sind an der BTU Cottbus noch nicht abschließend getroffen. Entscheidend ist allerdings, dass sich das IKMZ in diesen Zielfindungsprozess einbringt und mit seinem Dienstleistungsspektrum unmissverständlich verdeutlicht, dass sein Auftrag in Entlastung und Unterstützung der Fakultäten begründet liegt.

\section{$4 \quad$ Was wurde bisher erreicht?}

Nach einem guten Jahr konnten seit Gründung des IKMZ vor allem folgende Ziele erreicht werden:

- Festlegung der Dienste- und Servicestrukturen der Bibliothek im neuen IKMZ-Gebäude einschließlich des zur Gewährleistung des Servicespektrums notwendigen Personalkonzepts,

- konzeptionelle und räumliche Einbeziehung des Multimediazentrums in das neue Gebäude als Dienstleister für e-learning/Multimedia und als Innovationsoffice,

- Bildung des neuen Bereichs ,Betriebliche Datenverarbeitung', in dem der bisherige Bereich ,Verwaltungsdatenverarbeitung' mit der UB-Abteilung ,Bibliotheksdatenverarbeitung' zusammengeführt wurde,

- Entwicklung und Aufbau eines ,Corporate Design' für das IKMZ und einer entsprechenden WEB-Präsenz,

- Optimierung des Authentifizierungsdienstes für den Zugang zu Fest- und Funknetz (Single-Sign-On),

- Ausbau der Online-Zulassung und Online-Prüfungsanmeldung für Studierende sowie Vorbereitung der Einführung des HIS-Moduls LSF,

- Aufbau des Hochschul-Verbund-Multimedia-Brandenburg (HVMB) mit den Teilprojekten Content-Management, e-Learning-Plattformen, Videoconferencing, Autorenwerkzeuge u. a.,

- Konzeption für einen gemeinsam von Bibliothek und Multimediazentrum betriebenen Service zu Produktion, Nachweis, Verbreitung und Speicherung digitaler Dokumente.

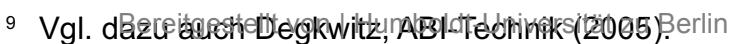


Mit diesen Maßnahmen sind Voraussetzungen geschaffen worden, die den anvisierten Integrationsprozess vorantreiben. Die damit erreichten Ergebnisse verstehen sich nicht als abgeschlossen, sondern als Einstieg in eine Entwicklung, die auf Konsolidierung, Kostenoptimierung und Kundenorientierung setzt. Der Schwerpunkt kann dabei - auch organisatorisch - nicht auf einer perfekten Lösung liegen, vielmehr steht ein guter Service im Mittelpunkt, der die Wettbewerbsfähigkeit der Forschungsund Studienbedingungen der BTU Cottbus effizient unterstützt. Die Herausforderung des Ansatzes liegt einerseits in der Entscheidungs- und Zielfindung zur Positionierung der Informationsinfrastruktur und andererseits in der Flexibilisierung der Organisationsabläufe, um die auf der Hochschulentwicklung gründenden Anforderungen an das IKMZ aufzugreifen und umsetzen zu können. Anders gesagt handelt es sich insbesondere für die erste und zweite Leitungsebene um einen Veränderungsprozess, der von fachspezifischen Problembehandlungen zu interdisziplinären Managementaufgaben führt, deren Erfüllung an der nutzerseitigen Akzeptanz des Diensteund Servicespektrums des IKMZ gemessen wird. Die weiterhin unerlässliche Fachkompetenz wird dabei verstärkt im Sinne der Hochschulentwicklung eingesetzt und ist ein entscheidender Faktor für den Erfolg. Insofern ist der Verzicht auf Perfektion und Wissenschaftlichkeit kein Verlust, sondern die entscheidende Chance, im Wettbewerb zu bestehen.

\section{$5 \quad$ Literatur}

Cowen, William A.: The Adsetts Center, Sheffield Hallam University. A case study of a multi-functional learning center. In: Library Quarterly 9 (1999) S. 39-57.
Beagle, Donald: Conceptualizing an Information Commons. In: The Journal of Academic Librarianship 25 (1999) S. 82-90.

Bulpitt, Graham: The Learning Center approach to elearning.<http://www.tu-dresden.de/t2002/bulpitt_ didni2002.pdf>.

Lütke-Entrup, Monika; Panke, Stefanie und Guy Tourlemain: Perspectives on ICT in German Higher Education. In: van der Wende, Marijk und Maarten van der Ven: ICT in Higher Education - A mirror of Europe. Utrecht 2003.

Roosendaal, Hans; Geurts, Peter und Eberhard Hilf: Pertinent Strategy Issues in Scientific Information and Communication <http://www.isn-oldenburg.de/ hilf/vortraege/saur04/ roosendaal-paper-final.pdf>. As printed version in: Bibliothekswissenschaft - quo vadis? München 2004.

Simon, Theresia: Die Positionierung einer Universitätsund Hochschulbibliothek in der Wissensgesellschaft. Eine bibliothekspolitische und strategische Betrachtung. Ravensburg 2004.

Simon, Theresia: Alternativen der strategischen Positionierung einer Universitätsbibliothek. In: ZfBB 51 (2004) S. 141-151.

Virkus, Sirje und Silvi Metsar: General introduction to the role of the library for university education. In: Library Quarterly 14 (2004).

Degkwitz, Andreas: <http://www.tu-cottbus.de/ikmz> - was heißt IKMZ? In: ABI-Technik 25 (2005) 1, S. 32-35.

Warnatz, Annette: Eine Medienburg aus Glas und Beton. In: BuB 57 (2005) Ausgabe 5, S. 349-354.

Degkwitz, Andreas: Das IKMZ der BTU Cottbus als ein eLearning-Resource-Center. In: B.I.T-Online 8 (2005) Ausgabe 2, S. 133-138.

$6 \quad$ Abbildungen

\section{INFORMATIONS-, KOMMUNIKATIONS- UND MEDIENZENTRUM (IKMZ)}

Dienstleistungsempfänger - „Kunden“ Professoren, Wissenschaftler, Studierende, Verwaltung, externe Nutzer, ...

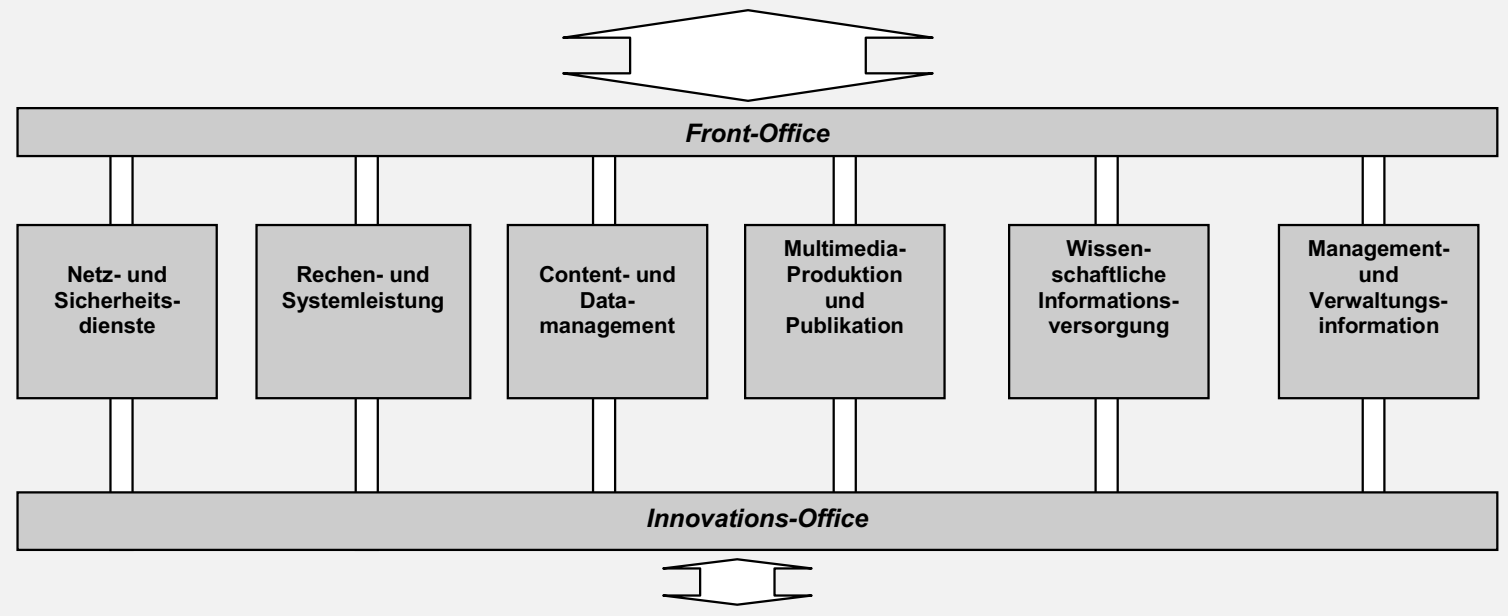

Forschungsbereiche 

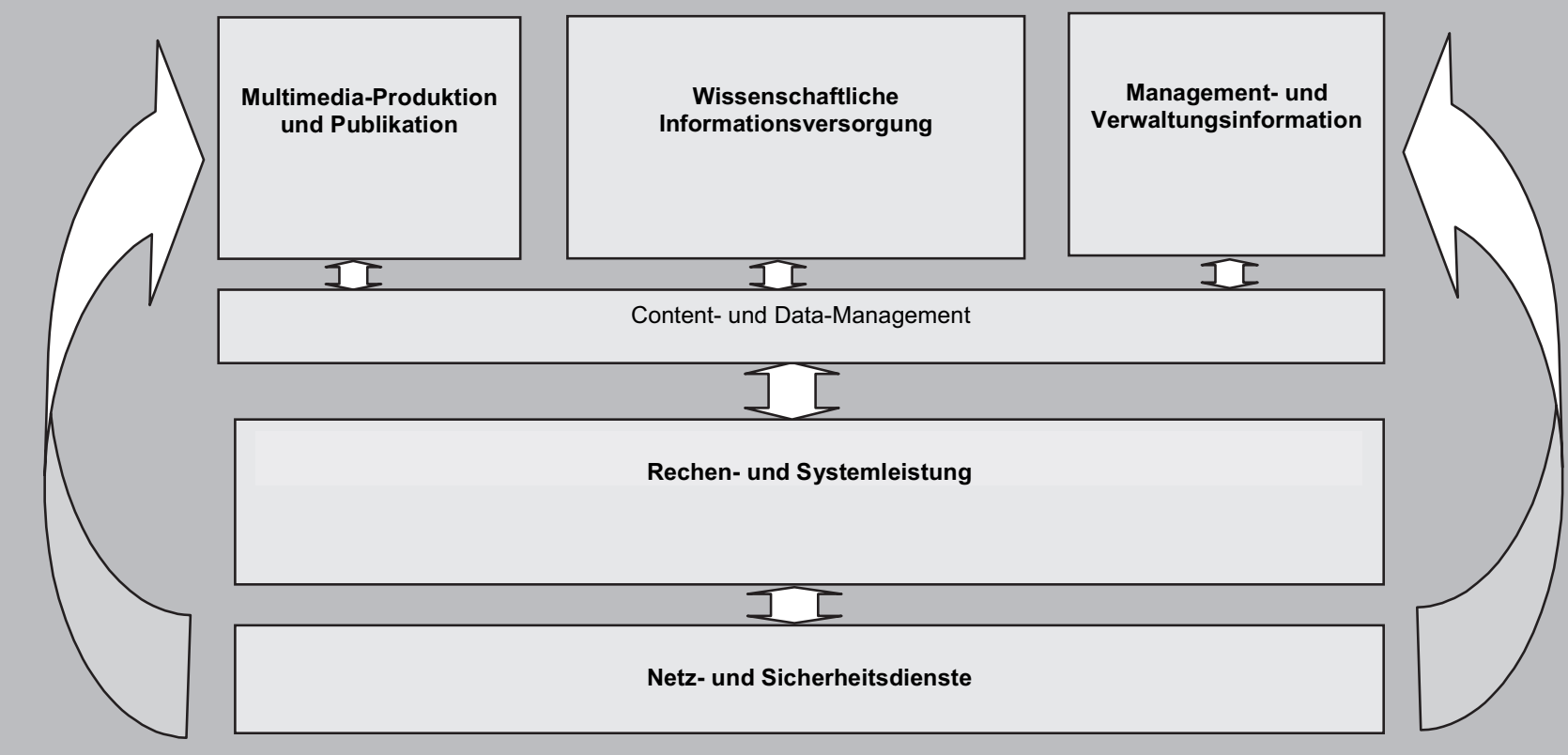

Abb. 2: Schichtenmodell der Aufgaben- und Funktionsbereiche

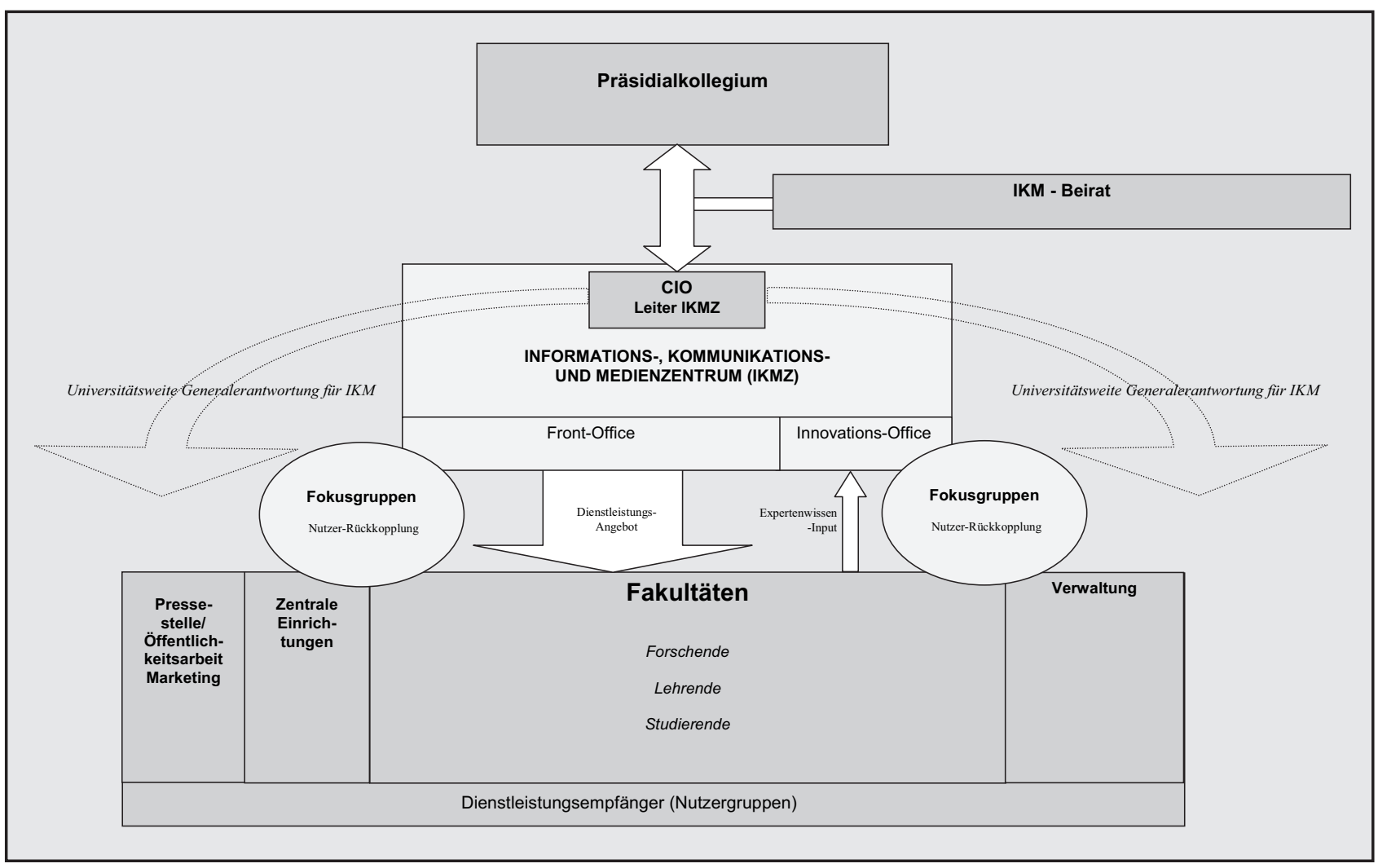

Abb. 3: Einbindung des IKMZ in die Struktur der Universität

\section{Anschrift des Autors:}

Dr. Andreas Degkwitz IKMZ der BTU Cottbus

Postfach 101344
D-03013 Cottbus

Tel.: ++49(0)355-69-2337

Fax: ++49(0)355-69-2277

E- Mail:Edegikwitz@ter-colttbus!deUniversität zu Berlin 